
cultural y científica entre Perú y Francia

\title{
Los franceses en el Perú en el siglo XIX: retrato de una emigración discreta
}

Les Français au Pérou au XIXe siècle : portrait d'une émigration discrète

French people in Peru in the 19th Century: portrait of a discreet emigration

\section{Pascal Riviale}

\section{OpenEdition}

Journals

\section{Edición electrónica}

URL: http://journals.openedition.org/bifea/4627

DOI: $10.4000 /$ bifea.4627

ISSN: 2076-5827

\section{Editor}

Institut Français d'Études Andines

\section{Edición impresa}

Fecha de publicación: 8 mayo 2007

Paginación: 109-121

ISSN: 0303-7495

Referencia electrónica

Pascal Riviale, «Los franceses en el Perú en el siglo XIX: retrato de una emigración discreta », Bulletin de l'Institut français d'études andines [En línea], 36 (1) | 2007, Publicado el 08 mayo 2007, consultado el 01 diciembre 2020. URL : http://journals.openedition.org/bifea/4627 ; DOI : https://doi.org/10.4000/ bifea.4627

Les contenus du Bulletin de l'Institut français d'études andines sont mis à disposition selon les termes de la licence Creative Commons Attribution - Pas d'Utilisation Commerciale - Pas de Modification 4.0 International. 


\title{
Los franceses en el Perú en el siglo XIX: retrato de una emigración discreta
}

\author{
Pascal Riviale*
}

\begin{abstract}
Resumen
Cuando se analizan los movimientos migratorios hacia el Perú en el siglo XIX, se puede difícilmente imaginar que los franceses constituyeron una de las colonias extranjeras más importantes en el país (la tercera colonia europea después de los italianos y los británicos según el censo de 1876). Por eso debemos constatar que representó una inmigración que se hizo poco notar. Este artículo se propone esbozar en sus grandes líneas el perfil de esta comunidad francesa del Perú, así como su evolución en el tiempo. Sin sorpresa mayor observamos (como en el caso de los demás destinos en América Latina) la predominancia de los inmigrantes oriundos de la parte suroeste de Francia (los Pirineos Atlánticos y la región de Burdeos). La proporción notable de migrantes declarando venir de la región parisiena, deja suponer que se podría tratar en este caso de una procedencia intermediaria representando una simple étapa desde una región inicial desconocida. Los franceses se ilustraron sobre todo en el comercio de detalle y de lujo, sin embargo son los pequeños artesanos quien constituyeron el sector profesional más numeroso de esta comunidad, que empezó a reducir progresivamente después de la Guerra del Pacífico.
\end{abstract}

Palabras clave: Perú, inmigración, siglo XIX

\section{Les Français au Pérou au XIXe siècle : portrait d'une émigration discrète}

\section{Résumé}

Lorsque l'on considère les mouvements migratoires vers le Pérou au XIXe siècle, on a peine à imaginer que les Français y représentaient l'une des plus importantes communautés étrangères (la troisième colonie européenne après les Italiens et les Britanniques selon le recensement de 1876). Force est de constater qu'il s'est agi d'une immigration qui se faisait assez peu remarquer. Cet article se propose donc de dessiner dans ses grandes lignes le profil de cette communauté française du Pérou, ainsi que son évolution dans le temps. Sans grande surprise, on constate (comme pour les autres destinations 
latino-américaines) la prédominance marquée des immigrants originaires du Sud-Ouest de la France (principalement les Pyrénées Atlantiques et le Bordelais). En revanche, la forte proportion d'individus déclarant venir de la région parisienne laisse supposer qu'il pourrait s'agir en fait d'une provenance intermédiaire, constituant une étape depuis une région initiale indéterminée. Si les Français se sont beaucoup investis dans le commerce de détail et de luxe, ce sont néanmoins les petits artisans qui constituaient le secteur professionnel le plus représenté de cette communauté, qui commença à décroître progressivement après la Guerre du Pacifique.

Mots clés : Pérou, immigration française, XIXe siècle

\title{
French people in Peru in the 19th Century: portrait of a discreet emigration
}

\begin{abstract}
When we talk about migratory movements to Peru in the $19^{\text {th }}$ Century, it is difficult to imagine that French people formed one of the most important foreign comunities there. They constituted the third largest European colony, after Italians and British people in the census of 1876 . Thus, we have to recognize it was a quite discreet inmigration. The purpose of this article is to describe the French community living in Peru, as well as its evolution in history. Not surprisingly, majority of migrants came from the southwest of France (Pyrénées Atlantiques and the region of Bordeaux). An important proportion of migrants declared as coming from Paris can maybe be understood as using Paris as an intermediary provenance, just a step in their move from an undetermined region of origin. French people were mostly involved in the small-scale and luxury commerce, but crafstmen formed a major sector of activity for this colony, whose size began to reduce after the war with Chile.
\end{abstract}

Key words: Peru, Inmigration, $19^{\text {th }}$ Century

Si los vínculos entre Francia y el Perú son regularmente sugeridos a través de la evocación de alguna personalidad que ha marcado de manera particular la historia peruana (por ejemplo, Auguste Dreyfus, Edmond de Lesseps, Eugène Courret, Léonce Angrand, el almirante Du Petit Thouars, etc.), en cambio nunca se hace mención a la presencia francesa en tanto que movimiento migratorio. Mientras que varias obras ya han sido consagradas a las principales comunidades extranjeras que han contribuido en la constitución de la nación peruana en el siglo XIX, no existía hasta el momento ningún estudio de síntesis referente a la comunidad francesa. Este texto — preludio de un libro en preparación-, se propone presentar a grandes rasgos un cuadro de esta emigración francesa al Perú en el siglo XIX, mostrando algunas de sus características, tal como aparecen a la luz de los registros de inscripción y de estado civil del Consulado de Francia en Lima. Para este estudio, se han completado dichas fuentes con diversas otras1 (observaciones de viajeros, censos hechos por el gobierno peruano, periódicos, etc.). Para comenzar presentaremos un panorama de la evolución de esta comunidad a lo largo

1 Es conveniente señalar por ejemplo que los registros de estado civil del consulado no reflejan necesariamente toda la vida de la comunidad francesa: muchas partidas de nacimiento, bautizo, matrimonio y defunción eran hechas en las parroquias. Teodoro Hampe Martínez ha elaborado una lista consecuente de estas partidas en un artículo consagrado a los archivos del Arzobispado de Lima (Hampe Martínez, 1993).. 
del siglo XIX, proponiendo algunos estimados numéricos tal como nos los entregan las fuentes disponibles; enseguida analizaremos las principales procedencias de origen de estos emigrantes. Por último evocaremos los principales sectores de actividad ocupados por estos últimos.

\section{PANORAMA HISTÓRICO DE LA PRESENCIA FRANCESA: ALGUNAS CIFRAS}

Algunos testimonios confirman la presencia aislada de algunos franceses en el Perú en los primeros años del periodo colonial: Jean-Pierre Tardieu (1995) recoge así el caso de algunos franceses acusados de herejía en el siglo XVII. Pero esta presencia es residual en extremo. En cambio, en el transcurso de las primeras décadas del siglo XVIII las circunstancias políticas y económicas iban a ocasionar un aflujo particularmente notorio de navíos franceses durante el periodo del contrabando malouin ${ }^{2}$ (entre 1700 y 1725). Los negociantes franceses efectuaban ya desde tiempo atrás un comercio indirecto con las Américas, por intermedio de sus homólogos españoles (incluso de franceses que habían tomado la nacionalidad española) establecidos en Cádiz. Sin embargo, la emergencia de un nuevo contexto geopolítico y económico iba a orientar a los comerciantes franceses hacia nuevas prácticas comerciales. Tras la creación de la Compañía de las Indias Orientales por Colbert (ministro de finanzas de Luis XIV) en 1664, numerosos negociantes estaban al acecho de inversiones fructíferas en materia de comercio con países lejanos. Si la apertura del comercio con China en los últimos años del siglo XVII ofrecía perspectivas interesantes, los informes hechos por filibusteros respecto de la riqueza de algunos puertos sudamericanos hacían soñar aún más. Desde ese entonces comenzaron a circular proyectos de comercio, incluso de implantación de colonias francesas sobre las costas de Chile y del Perús. Así es como un negociante de Saint-Malo, Noël Danycan, se asoció con el comerciante parisino Jean Jourdan, para organizar una expedición marítima con destino a las costas americanas del Pacífico con el objetivo de comprobar el interés que habría en explotar estos nuevos mercados comerciales. Para este efecto se fundó una «Compañía del Mar del Sur» en el verano de 1698. Una primera expedición, efectuada entre 1699 y 1701, dejó entrever las posibilidades comerciales de estas colonias españolas en la búsqueda de nuevos productos manufacturados. Después de algunas dudas y de nuevas exploraciones comerciales hacia los mares del sur, a partir de 1703 los negociantes malouins quedaron convencidos del potencial financiero de estas operaciones. Desde entonces un número creciente de navíos iba a ser armado para dirigirse al Pacífico tomando una nueva ruta transoceánica pasando por el Cabo de Hornos, aprovechando en primer lugar de los disturbios y de la incertidumbre jurídica ocasionados por la Guerra de Sucesión de España para desarrollar un comercio intérlope con las colonias de la costa del Pacífico de América del Sur.

En el espacio de unos cuantos años los intercambios comerciales fueron tan intensos en esta zona que los negociantes franceses llegaron incluso a hacer construir almacenes en los puertos más ventajosos para su tráfico con el fin de almacenar sus mercaderías, pero también casas para alojar a sus tripulantes que venían a enfrentarse con los rudos elementos del hemisferio sur. En su estudio relativo a los negociantes malouins André Lespagnol describe la situación particularmente esclarecedora de este informe de Concepción hacia 1700:

«Con el tiempo, la multiplicación del paso de navíos franceses, el alargamiento de la duración de las escalas, puesto que muchos navíos efectuaban allí una verdadera invernada durante varios meses, multiplicándose también las deserciones —que un capitán evaluaba en cerca de 2000 desde 1708- se desarrolló en esta zona un verdadero proceso de semi-sedentarización». (Lespagnol, 1997, II: 602)0

2 De Saint-Malo, puerto de la costa atlántica francesa (nota de la traductora).

3 Uno de esos primeros proyectos sería atribuible a un oficial de la Marina Real, M. de Gennes, en los últimos años del siglo XVII (Dahlgren, 1909: 113) 
Por cierto, Concepción ocupaba una posición de excepcional envergadura en la estrategia comercial de los negociantes franceses pero estos últimos habían transpuesto en menor medida un modelo similar en algunos puertos intermedios peruanos tales como Arica, llo o Pisco. En espera de la llegada de los comerciantes de Lima o bien del Alto Perú, los franceses habían construido tiendas y viviendas precarias ${ }^{4}$. André Lespagnol observa además que un número importante de súbditos franceses que habían decidido establecerse allí sirvieron de intermediarios entre negociantes franceses y peruanos (Lespagnol, 1997: 612). Si es difícil cifrar en forma precisa el número de franceses que abordaron las costas del Perú, podemos citar, para dar una idea de la importancia del tráfico intérlope, la evaluación hecha por André Lespagnol: este autor ha registrado un mínimo de 137 navíos franceses armados con destino a los mares del sur entre 1698 y 17245 . Empero, este flujo marítimo iba a tender a reducirse considerablemente a partir de 1715 por el hecho de la presencia excesiva de navíos franceses y como consecuencia de una saturación de los mercados locales. Además, una política más represiva del Virrey terminó convenciendo a los inversores de la inutilidad de continuar en estas empresas de contrabando. Aunque las autoridades coloniales ordenaron enseguida la expulsión de los comerciantes franceses aún instalados en el Perú, parecería sin embargo que un número considerable de marinos y de artesanos franceses obtuvo la autorización de permanecer en él seguramente porque sus capacidades profesionales y sus conocimientos debieron ser juzgados útiles para la actividad económica local. Después, en el transcurso del siglo XVIII, otras llegadas más puntuales mantuvieron esta presencia francesa —que volvió a ser muy marginal—. Una serie de investigaciones efectuadas durante el año 1776 culminó con la identificación de 163 extranjeros. Si bien se contaba entre ellos a una mayoría de portugueses y de italianos, había no menos de 31 franceses (Campbell, 1972: 156). Un observador informaba que en Lima los restaurantes y expendios de bebidas estaban en ese entonces exclusivamente en manos de italianos y de franceses. Los otros súbditos franceses eran artesanos y comerciantes. Por último, es interesante que otras fuentes hayan notado la presencia de franceses involucrados en actividades mineras en Huancavelica así como la de tres médicos ejerciendo en diferentes puntos de la provincia (Campbell, 1972: 162-163).

Tras la abdicación de Napoleón I y con la restauración de la monarquía un gran número de oficiales del antiguo Ejército Imperial fueron separados de las filas del ejército y puestos a media paga. Muchos de ellos prefirieron intentar una nueva vida en otro sitio en vez de vegetar esperando una hipotética reintegración. Es así como algunos llegaron a América del Sur y formaron parte de diferentes ejércitos libertadores opuestos a los españoles, en La Plata, en Colombia, en Chile, Perú, etc. Actor de la Independencia, el capitán Gabriel Lafond de Lurcy mencionaba a algunas de estas personalidades que tuvo ocasión de conocer durante sus estadías en el Perú: entre ellos citemos al general de brigada Frédéric de Brandzen, al general de brigada Eugène Giroust, a los coroneles Pierre Raulet, Alex Bruix, Albert d'Albes, a los mayores Jacques Mollet y Louis Soulanges, Sauveur Soyez, comisario de marina, a los capitanes de navío Guillaume Prunier e Hippolite Bouchard (oficiales del Estado Mayor de la primera flota del Perú independiente) (Lafond, 1844, vol. II: 281, 285, 292)6. Estos oficiales, convertidos en notables, iban a conformar junto con algunos negociantes el núcleo inicial de la colonia francesa de Lima. En ese entonces, esta se limitaba a algunas centenas de personas a las que se puede añadir algunos individuos dispersos en provincias. En su diario, Heinrich Witt menciona a dos negociantes franceses presentes en Arequipa en 1824 (Jacques Le Bris y M. Ponsignon), a M. de Romainville, a quien conoció cerca de Caracoto, a un tal Dupin que vivía en Cerro de Pasco en 1827 (Witt, 1992, I:

4 A su paso por llo en 1715, poco después de un sismo, un viajero francés observaba que buen número de estas tiendas se había derrumbado (de La Barbinais, 1728, vol. I: 67).

5133 navíos de comercio (de los cuales 115 tan solo para el periodo 1701-1716) y 4 navíos de la Marina Real (Lespagnol, 1997: 551).

6 Un pequeño informe intitulado La colonia francesa da algunos nombres y detalles adicionales (Sociedad Francesa de Beneficencia, 2002: 234-236). 
60 y 107); mientras que Lafond de Lurcy evocaba un viaje a Cajamarca realizado en 1827 en compañía de M. Condamin y del Dr. Martin, ambos asentados en Trujillo (Lafond, 1844, II: 415). Otros testimonios podrían completar seguramente este cuadro, el mismo que de todas maneras se refiere solo a algunas individualidades aún muy aisladas (Barrantes, 2005). Un informe del capitán de fragata Alphonse de Mogel (fechado el $1^{\circ}$ de diciembre de 1825) nos da una idea de la escasez numérica pero también económica de esta comunidad francesa:

«Hay pocas localidades importantes en el Perú, como en otros lugares de la Tierra, en donde no se encuentren algunos franceses. Pero con frecuencia no es la elite de la nación la que se va a vivir a lo lejos de una vida oscura. Puede que haya unos 300 franceses, generalmente artesanos en una situación poco acomodada o muy secundaria. Se observa una casa comercial francesa pero apenas acaba de establecerse» ${ }^{7}$. (PellegrinDrillet et cie)

Tras la Independencia, se habían desintegrado los grupos tradicionales de comerciantes españoles y criollos que monopolizaron el negocio durante la Colonia. Los negociantes europeos (principalmente anglosajones) aprovecharon esta oportunidad y ocuparon los vacíos así creados (Gootenberg, 1990: 239). Como testimoniaba el capitán de Mogel, los franceses estaban aún poco presentes en estos mercados y se encontraban sobre todo limitados a pequeños oficios. Las considerables ganancias producidas por la exportación de guano a partir de fines de los años 1830 y sobre todo de inicios de los años 1840 iban a provocar importantes cambios en la economía y en la sociedad peruana. El enriquecimiento de una elite comerciante iba a modificar el modo de vida y las costumbres de consumo de una parte creciente de la población (gusto por nuevos productos, más en el estilo de lo que se hacía en la Europa «moderna»), mientras que los gobiernos sucesivos se lanzaban en ambiciosos proyectos de modernización de las infraestructuras industriales y urbanas (instalaciones portuarias, construcción de puentes y de ferrocarriles, etc.) y de su sistema administrativo, educativo, de salud, etc. Tantas nuevas prácticas y proyectos que iban a atraer un número considerable de extranjeros.

En el espacio de algunas décadas esta fuerte dinámica económica iba a motivar una inmigración importante, particularmente de europeos que venían a proponer sus servicios o a buscar fortuna. Además de artesanos y negociantes atraídos por una coyuntura económica favorable, fuente de un posible éxito social de difícil acceso en sus países, numerosos técnicos, ingenieros y especialistas de todo tipo fueron contratados también por el gobierno peruano (o por hacendados) para ejecutar las modernizaciones proyectadas en el transcurso de este periodo fasto. Si a veces esta inmigración fue organizada a instigación del gobierno (a veces más o menos sin su consentimiento), como en el caso de la importación de culíes chinos o de diversos proyectos para hacer venir una mano de obra europea ${ }^{8}$, en los casos más frecuentes se trataba de una emigración voluntaria e individual. Es así como hay que entender esta llegada progresiva de franceses deseosos de encontrar un mejor futuro en suelo peruano. Esas cuantas cifras de las que disponemos permiten dibujar en grandes líneas la evolución numérica de esta colonia francesa y su distribución sobre el territorio peruano.

Hemos visto ya que, según la estimación del capitán de navío De Mogel, la comunidad francesa residente en Lima en 1825 podía estar formada por alrededor de 300 personas (probablemente en su mayoría hombres, a los que habría que añadir algunas decenas de individuos dispersos

7 Capitán de fragata Alphonse de Mogel: «Remarques sur quelques parties de l'Amérique du Sud. 1 ère partie : Pérou (Paris, $1^{\text {er }}$ décembre 1825) »: 89-90. Archives nationales, Paris. $F^{17} 7161$ (rapports commerciaux. Pérou 18251906).

8 A lo largo de toda la segunda mitad del siglo XIX se concibieron diversos proyectos que indican hasta qué punto era fuerte esta idea según la cual la única solución para modernizar el país era introducir en él mano de obra europea, supuestamente más competente (según criterios muy idealizados) o más apta para responder a una demanda específica de los empleadores. La ausencia de medios generalmente puestos a disposición de los candidatos a la emigración, la falta de organización de los planes concebidos, la dificultad de adaptación a medios a veces difíciles (como sucedió en el oriente peruano) acabaron frecuentemente en un fracaso dramático de estas inmigraciones organizadas oficialmente. 
en el conjunto del territorio peruano). El censo de los extranjeros asentado en 1840, aunque muy parcelario, nos proporciona algunas indicaciones interesantes sobre la distribución de esta población, por nacionalidad y por región de residencia. Aquel año, se censaron 1025 extranjeros sobre el territorio nacional (sin tener en cuenta a la capital - aparentemente excluida del censo o habiendo sido objeto de una contabilidad aparte y cuyos rastros no se han encontrado-). Entre aquellos se contaban 64 franceses, instalados principalmente en los departamentos de Moquegua (22), de Junín (9), de Ancash (8) y en El Callao (12)9. Esta relativa concentración se articula alrededor de grandes polos de actividad económica del Perú de esa época, a saber, las minas (Junín y Ancash), la explotación de nitratos (Moquegua), el comercio y el tráfico marítimo (El Callao). No obstante, es conveniente observar que los franceses censados en cada uno de estos departamentos no se dedicaban necesariamente a las actividades predilectas de estos departamentos respectivos: en realidad, cuando se precisa la profesión, esta corresponde con mayor frecuencia a actividades ligadas al pequeño comercio y a la artesanía. El pico de la inmigración europea corresponde a mediados del siglo XIX. El censo hecho en Lima en 1857 por Manuel A. Fuentes confirma este aflujo migratorio10: en ese año, tan solo en la capital se contabilizan 2693 franceses (sobre un total de 21557 extranjeros, es decir 12,5 \% de este total). Hay un hecho interesante y es que sobre estas 2693 personas se contaba a 595 mujeres, prueba que esta inmigración no era solamente masculina sino que emigraban familias enteras. Entre esta gente femenina se encontraban también mujeres solas (viudas o solteras) que habían venido a buscar una vida mejor a orillas del Pacífico. Un nuevo censo efectuado en provincias en 1860 viene a corroborar este desarrollo de la colonia francesa, ilustrándonos sobre su distribución espacial en el país. No son menos de 133 los franceses registrados entonces en el departamento de Moquegua (en ese momento en plena explotación de nitratos), mientras que se cuentan 40 en el departamento de Junín (entre los cuales 16 mineros). Vienen después la provincia litoral de Ica (21), los departamentos de Ancash (16), de Arequipa (14), de Piura (10) y de Cuzco (8)11. Sin embargo, estas cifras no se pueden considerar con una confianza absoluta; falta la información relativa a algunos puntos de la república y cuando se nos las da, no toman en cuenta más que a la población masculina (los jefes de familia). Ejemplo de ello es el censo de extranjeros asentados en el departamento de Moquegua, en 1866. Este documento registra, calle por calle, a los residentes de las diferentes localidades del departamento (Tacna, Arica, Iquique, Mejillones, Ilo, etc.), su nacionalidad, su tiempo de residencia en el país y en la localidad, su profesión. Se alcanza un total de 168 hombres adultos, 35 mujeres y 35 niños (entre los cuales 11 nacidos en Francia), es decir 70 adultos más que en 1860. Ya sea que esto corresponda a una real progresión cuantitativa o simplemente a una mayor precisión de la encuesta, en todo caso, lo que esta última confirma es la importancia de este polo de actividades económicas que atraía en ese entonces a gran número de extranjeros. Es conveniente observar también que a partir de esta época la comunidad francesa iba a comenzar a organizarse formalmente, a través de diferentes asociaciones de ayuda mutua y de diversión, índice adicional de una mutación en curso en su relación con la sociedad peruana.

El primer cuadro general de la comunidad francesa del Perú data de 1872, cuando M. de Saint-Quentin, encargado de negocios de Francia en el Perú dirigió al Ministerio de Asuntos Extranjeros, en Francia, un «estado numérico de los franceses domiciliados en el Perú al mes de diciembre de 1872»12. Si bien las localidades más frecuentadas eran como siempre Lima (1 861 residentes, de los cuales 478 mujeres y 160 niños) y El Callao (275, de los cuales 61 mujeres y

9 «Razón de los extranjeros...» Archivo del Ministerio de Relaciones Exteriores. 2 =. E.: Prefecturas (1840). Para una síntesis de este censo (y también del de 1860) ver Hünefeldt, 1987, tomo I: 141-196.

10 Ver análisis del censo de 1857 en Bonfiglio (1987: 31-78) y su cuadro $\mathrm{n}^{\circ} 4$. Un análisis más completo de los movimientos migratorios europeos hacia el Perú ha sido recientemente publicado por Bonfiglio; en él se encuentra este cuadro y muchas otras fuentes útiles (Bonfiglio, 2001: 13-164).

11 Archivo del Ministerio de Relaciones Exteriores. 2 O. E.: prefecturas (1860). Obsérvese que no se encontraron los datos del Callao y del departamento de La Libertad.

12 Archivos del Ministerio de Asuntos Extranjeros, París. Correspondencia consular. Perú 35 (1873). 
67 niños), otras ciudades mostraban una concentración no observada hasta entonces. Es el caso de Trujillo, en donde el diplomático manifestaba la presencia de 126 franceses, o de Arequipa (con 99 residentes de origen francés). El número total de franceses que habitaban en el Perú era estimado en ese entonces en 2625 ( 1734 hombres, 586 mujeres y 305 niños). Por propia confesión del diplomático que había hecho este cuadro estadístico, el resultado de su trabajo era incierto y probablemente subevaluado:

«Esta cifra no es más que aproximativa y me lleva a pensar que está por debajo de la verdad. En efecto, en los alrededores de la capital y de las principales ciudades de la república e incluso en el campo existe un cierto número de franceses empleados en las labores de las haciendas, y que es imposible determinar. Hay también quienes por diversos motivos prefieren permanecer sin ser conocidos. Me parece pues que se podría, sin exagerar, llevar a 3000 el total de nuestros nacionales en el Perú»13.

El censo nacional hecho en 1876, bajo la dirección de Manuel Atanasio Fuentes, llegó a un resultado casi similar: en suelo peruano habían 2658 individuos de nacionalidad francesa (1 935 hombres, 723 mujeres), aunque en algunos departamentos sean difíciles de explicar las diferencias numéricas que aparecen a la lectura de estos dos documentos. Así como la estimación hecha por el diplomático francés en 1872 está sin duda alguna por debajo de la verdad, se sabe que el censo de 1876 está manchado por algunos errores o aproximaciones ${ }^{14}$ : así, las lagunas existentes en las informaciones transmitidas por algunos agentes censadores terminaron imposibilitando la identificación de la nacionalidad de 5184 personas reconocidas como extranjeras (Bonfiglio, 1987: 42). Para hacerse una idea de la gran incertidumbre relativa a estas evaluaciones cifradas, veremos que cuando en 1876 Manuel Fuentes daba para Lima la cifra de 1531 residentes de nacionalidad francesa, un informe del ministro plenipotenciario de Francia en Lima (Domet de Vorges), fechado en julio de 1880, informaba acerca de los temores de las comunidades extranjeras frente a la cercanía de las tropas chilenas y sobre las posibles consecuencias de los combates, y que por ello el diplomático alentaba a sus compatriotas a inscribirse en el consulado para poder estar en medida de garantizar su neutralidad, y señalaba:

«Aprovecho para hacer inscribir a un gran número de franceses que no habían cumplido nunca con este trámite. El número de individuos inscritos sube, en este momento, a más de 1 100, lo que, teniendo en cuenta a las mujeres, a los niños y a las personas que no se habían presentado todavía, debe llevar a más de 3000 individuos a la colonia francesa de Lima»15.

De hecho, muchas personas no consideraban útil hacerse inscribir en el consulado16, hasta preferían evitarlo. Hay que saber que un número importante de jóvenes se exilaban para evitar la conscripción; el fenómeno es particularmente conocido en cuanto al país vasco pero, por cierto, no solo se lo observa allí. Sin duda había muchos otros motivos para no hacer los trámites ante el consulado. En consecuencia, queda claro para nosotros que estos registros de inscripción son tan solo una fuente fragmentaria: solo aparecen aquellos que quisieron presentarse. Es necesario entonces contar con otros tipos de fuentes para completar las informaciones disponibles.

Hacen falta igualmente las fuentes para estimar cuál era la parte de la población extranjera en el Perú de fines del siglo XIX, sin embargo, parecería que las dificultades económicas ${ }^{17}$ y la

13 Carta de Saint-Quentin al ministro de Asuntos Extranjeros. Archivos del Ministerio de Asuntos Extranjeros, París (Correspondencia consular. Perú 35, 1873: 8).

14 Además, existe falta de certeza en cuanto al censo de los niños.

15 Carta de Domet de Vorges al ministro Freycinet (Lima, 25 de julio de 1880). Archivos del Ministerio de Asuntos Extranjeros, París (Correspondencia consular. Perú 38, 1879-1880: 346).

16 Las inscripciones efectuadas en 1880 muestran que un gran número de estos «recién inscritos» residían a veces desde mucho tiempo atrás en el Perú. Tal es el caso del negociante Félix Dibos, que aunque residiendo en Lima desde 1858 no consideró útil — por motivos desconocidos— de matricularse en el Consulado antes de 1880 (Riviale, 2005).

17 Debidas en particular a la inmensa deuda proveniente del contrato Dreyfus relativo al guano así como a las consecuencias de la guerra con Chile (recesión económica, pérdida de los recursos de los nitratos, etc.). 
inestabilidad política del país después de la Guerra del Pacífico aminoraron no solo el interés de los candidatos a la emigración hacia esta posible tierra de acogida sino que también provocaron la partida de cierto número de extranjeros residentes en el Perú. Este fue aparentemente el caso de negociantes y de industriales. Como signo de esta regresión de la presencia francesa, en 1905 solo se censaron 167 franceses en El Callao (contra 375 en 1876) y en 1908 solo eran 872 en Lima (contra 1479 en 1876)18. Esta restricción numérica iba incluso a confirmarse en el curso de las décadas siguientes pues en 1920 se contaban 737 franceses en Lima y 111 en El Callao (Bonfiglio, 2001: anexos 6 y 11).

\section{LUGARES DE ORIGEN DE LOS MIGRANTES}

A pesar de todas sus imperfecciones, estos diferentes documentos nos dan por lo menos una idea de la importancia numérica de esta comunidad y de su evolución a lo largo del siglo. Luego parece interesante determinar cuáles eran los principales lugares de origen de aquella. Los registros de inscripción del Consulado de Francia constituyen la fuente principal de la que disponemos para obtener un panorama de la procedencia geográfica de los inmigrantes. Con el objetivo de reforzar esta base de datos hemos unido a ella las informaciones contenidas en los registros de estado civil, lo que nos ha permitido llegar a tener una base de datos con 4000 nombres, a partir de la cual son posibles diversos análisis estadísticos.

Esta base, sin ser exhaustiva por cierto, parece suficientemente representativa por lo menos de la población masculina presente en el Perú en un momento dado. En lo referente a la procedencia, se puede determinar así que los departamentos franceses de origen más representados son los siguientes: Gironda (12\% del total), Sena (9\%), Pirineos Atlánticos $(9 \%)$, y muy por detrás: Alta Garona (4 \%), Lot (3\%), Bajo Rin (2 \%), Altos Pirineos (2 \%), Bocas del Ródano (1 \%), Loira Atlántico (1\%) y Ródano (1\%).

En su estudio sobre la emigración desde el puerto de Burdeos, Philippe Roudié destaca el hecho que, mezclando todos los destinos, los migrantes provenientes de los departamentos de los Pirineos Atlánticos y de los Altos Pirineos ocupan los dos primeros lugares, delante de los girondinos (Roudié, 1983: 178). Es interesante observar en el caso de los emigrantes hacia el Perú la predominancia de las personas que declararon como su lugar de origen al departamento de Gironda: aquí este se encuentra por delante del departamento de los Pirineos Atlánticos. En efecto, los primeros en haber emigrado en masa hacia este país son gentes originarias de la región de Burdeos: representaban $14 \%$ de las personas inscritas en el Consulado de Francia en Lima en el periodo 1840-1844, $27 \%$ en 1845-1849 y más de $16 \%$ de los inscritos entre 1850-1854. Después la proporción pasó a ser menos marcada, oscilando entre 7,5 \% y $10 \%$ entre 1855 y 1874, y bajando hasta un $5 \%$ del total en el último cuarto del siglo XIX. Entre estos girondinos se encontraban, mezclando todos los periodos, un número particularmente considerable de comerciantes. La importancia del número de individuos que se calificaban de «negociantes» parece indicar que así se desmarcaban de los minoristas y tenían más que ver con negocios de mayor escala impulsados desde Burdeos (sin duda alguna muchos de ellos eran representantes de casas comerciales establecidas en Burdeos). Son conocidos los importantes contactos comerciales que unían al puerto ${ }^{19}$ de Burdeos (y más ampliamente a la región de Burdeos) con América del Sur. Sin embargo, es conveniente observar que el número de artesanos inscritos en el consulado es aún ligeramente superior al de los individuos entregados a las actividades comerciales (32 y $31 \%$ respectivamente). Sin embargo, la importancia considerable del contingente de personas originarias del departamento de Gironda debe ser analizada con precaución. La mención «lugar de origen» tal como aparece en los registros de inscripción del Consulado de Francia podría

18 Censo del Callao, 1905 y Censo de la Provincia de Lima, 1908 en Bonfiglio, 1987 (cuadros 3, 14 y 15 ).

19 El número de marinos oriundos de Burdeos (particularmente en la primera mitad del siglo XIX) que aparecen en los registros del consulado es un indicador adicional de estas relaciones portuarias. 
inducirnos a error: en efecto, es posible que algunos recién llegados hayan declarado Burdeos como lugar de origen pensando en su último lugar de residencia (o lugar de emisión de su pasaporte) y no refiriéndose a su lugar de nacimiento. Como Burdeos fue el principal lugar de emigración hacia América del Sur durante gran parte del siglo XIX, es obvio que la mayoría de las personas que llegaron al Perú hayan transitado por esta ciudad.

El segundo grupo en importancia numérica es el de las personas oriundas del departamento de los Pirineos Atlánticos que fue tradicional en la emigración francesa hacia América Latina; se conoce el rol considerable desempeñado por los vascos en la colonización de la región de La Plata y, más ampliamente, de todo el Cono Sur, desde inicios del siglo XIX hasta mediados del siglo XX. Entre 1835 y 1842 Uruguay habría así atraído a 13765 inmigrantes franceses, en su mayoría oriundos del departamento de los Pirineos Atlánticos, mientras que en 1860 se contaba ya con 40000 vascos y bearneses en Argentina (Charnisay, 1996: 198-199)20. Chile también atrajo a varios miles de individuos 21 , entre los cuales a un número considerable de girondinos y de vascos que, en el caso de algunos, echaron raíces en el lugar (Blancpain, 1999: 134135). La imagen tradicional (dada por ejemplo por Charnisay, 1996) nos muestra efectivamente a migrantes originarios del departamento de los Pirineos Atlánticos que serían, por un lado, obreros, artesanos, jornaleros y pequeños propietarios que partían con su familia, abandonando su país como consecuencia de dificultades económicas coyunturales (malas cosechas y ruina de la pequeña artesanía frente al desarrollo de la industria), y por otro lado, hijos no primogénitos de familias campesinas o burguesas que partían para escapar de la conscripción o para buscar un futuro mejor en un mundo nuevo22. Si este esquema se corrobora particularmente en los países del Cono Sur, es solo verdad en parte en el caso del Perú en donde las actividades agrícolas o ganaderas no movilizaron una mano de obra tan considerable: si como sus compatriotas que partieron a la Argentina o al Uruguay, los vascos y bearneses establecidos en el Perú no tenían necesariamente muchos medios, a menudo se tornaron hacia actividades artesanales urbanas, o se pusieron al servicio de algún pariente, amigo o compatriota. La parte importante de empleados (en comparación con lo que se constata en el caso de Aquitania) se explica sin duda a la luz de este contexto particular. Si una proporción importante de individuos oriundos del País Vasco había optado por emigrar (particularmente a América del Sur) para dejar atrás una situación local difícil, este motivo es lejos de ser el único, cierto número de ellos se integraron ciertamente en una red de parentela o de relaciones que los había llevado a dejar Francia para establecerse en América del Sur, sin duda ya con un proyecto definido o por lo menos con suficientes datos como para no partir hacia algo totalmente desconocido y hacia un futuro incierto. Las cifras proporcionadas por los registros de inscripción del consulado ilustran claramente acerca de la situación de los emigrantes originarios del departamento de los Pirineos Atlánticos que vinieron a asentarse al Perú. Se observa una mayoría muy grande de comerciantes $(34,5 \%)$ y de artesanos ( $25 \%$ ), a la que hay que sumar un número considerable de «empleados» (en el comercio o en un taller) y de personas dedicadas a las actividades hoteleras y de restaurantes (más del $5 \%$ ). Por último, notemos también el porcentaje importante de individuos provenientes del sector agrícola (cerca del $5 \%$ ) -imagen del origen rural de estos vascos que se ilustraron en otras partes en la colonización y la explotación de las tierras baldías de América del Sur-.

Es conocida la importancia del rol desempeñado por las agencias de emigración en el País Vasco para atraer a los candidatos a la emigración. Estas agencias actuaban esencialmente a solicitud

20 Aunque de reciente publicación, se trata en realidad de una obra escrita en 1947 cuyas estadísticas lamentablemente se caracterizan por tener muchas lagunas. Guy Bordé, por su lado, indica que entre 1857 y 1927 habrían ingresado a Argentina, con la intención de asentarse allí 226000 franceses, en su mayoría vascos o bearneses (Bourdé, 1974: 169).

21 Las cifras oficiales (1 654 franceses en 1854, 3192 en 1875, 8266 en 1895 y 9800 en 1907) son sin duda claramente inferiores a la realidad (Blancpain 1999: 130-131).

22 El aún vigente derecho de primogenitura dejaba en efecto a numerosos hermanos menores $\sin$ recursos y $\sin$ perspectivas de futuro (Charnisay, 1996: 115 y 150). 
de los gobiernos de Argentina, Uruguay, Paraguay y — sin duda en menor medida-Chile. No es nada seguro que haya ocurrido lo mismo en lo que atañe al Perú. La escasa atracción de los emigrantes hacia este país no alentó seguramente el interés de los agentes de emigración para trabajar este destino. En este caso, es más probable que tengamos ante nosotros una emigración motivada por el éxito de algunos «pioneros», el mismo que debió atraer a miembros de la familia ampliada, del grupo de amigos, de las personas oriundas del mismo cantón. Tuvo que funcionar la solidaridad para ayudar a los recién llegados a instalarse23; solidaridad que encontramos en los registros del consulado: así, con motivo del deceso de un tal Junquet en 1868 sirvieron de testigos siete personas originarias del departamento de los Pirineos Atlánticos; o también con ocasión de la boda de Michel Sallaberry con Gracienne Larrabure en 1865, encontramos entre los testigos los nombres de otros vascos como Bidegaray, Athano, Otheguy, Gaillour24.

La aplastante proporción de migrantes originarios del suroeste de Francia se ve reforzada por la contribución de otros departamentos representados a menudo en otras partes de América Latina: Alto Garona, Lot, Altos Pirineos. Cabe notar además que la recurrencia de algunas localidades permite suponer redes de inmigración, sin duda totalmente informales y puntuales, pero que ilustran micro fenómenos muy reales. En varios periodos del siglo XIX se hace así mención a pequeños pueblos del cantón de Saint-Gaudens (por ejemplo, Labarthe-Rivière, Pointis-deRivière o Labroquère). Es el caso de diferentes personas que llevan los apellidos Save o Save de Pujolle, originarios de Pointis-de-Rivière así como de aquella familia de carniceros de apellido Betmal (o Betmalle), aparentemente oriundos todos del pueblo de Labroquère; a lo largo de los años todas estas personas aparecen en los registros de inscripción del consulado. En definitiva, toda esta región pirenaica constituye una zona fuerte de emigración. Rolande Bonnain, en su estudio de caso, observa esta misma y clara presencia pirenaica (con lazos de parentesco en los mecanismos migratorios) en Uruguay y Venezuela (Bonnain, 2000).

La muy alta proporción de inmigrantes franceses que declararon al departamento del Sena como lugar de origen no tiene que sorprendernos aún cuando la interpretación de esta constatación sea incierta. París y su región presentaban una densidad poblacional que puede en sí justificar esta alta presencia en el seno de la comunidad francesa del Perú. Todos estos individuos no eran quizá tampoco parisinos de origen: desde las primeras décadas del siglo XIX esta capital ejercía un evidente poder de atracción sobre los provincianos (a menudo pequeños artesanos y gente del mundo rural) sin trabajo que esperaban encontrar en ella un empleo frecuentemente difícil de encontrar en su tierra natal. En este caso París podría ser percibido como una etapa intermedia en el recorrido de provincianos que intentaron asentarse en la capital francesa y luego decidieron intentar suerte en el extranjero.

El último caso que trataremos aquí será el de los alsacianos cuyo número es relativamente considerable entre los inmigrantes al Perú (cerca de 2,5\% del total). La tradición migratoria de los departamentos del Bajo Rin y del Alto Rin hacia las Américas (sobre todo a América del Norte desde luego) parece remontarse al siglo XVIII en el que individuos nativos de esta región del este de Francia habrían seguido a los importantes contingentes de protestantes y de judíos alemanes que partieron a refugiarse a América huyendo de las persecuciones sufridas en su país. En el siglo XIX, los disturbios políticos y las dificultades económicas de la región alentaron este movimiento migratorio, el mismo que se acentuó aún más después de la derrota de Francia frente a Prusia en 1870. Alsacia y Lorena fueron cedidas al nuevo imperio germánico en 1871 y se pidió a sus habitantes optar ora por la nacionalidad francesa, ora por la nacionalidad alemana. Muchos de ellos prefirieron seguir siendo franceses 25 y se exiliaron en las Américas.

23 El considerable número de empleados podría ser un indicador de esta solidaridad que llevaba a una persona ya bien instalada a tomar como empleado a un joven o a un recién llegado oriundo de su mismo terruño.

24 Varios de ellos también se habían inscrito juntos en el consulado en 1858. Se constata además que algunos estaban igualmente ligados por matrimonio (un Gaillour casado con una Larrabure).

25 De los 80 individuos oriundos del Bajo Rin que aparecen en los registros del Consulado de Francia entre 1840 y 1895, 18 llegaron (o son mencionados por primera vez) en 1872 y 7 en 1873. Cabe notar que en 1872 los 


\section{LOS PRINCIPALES SECTORES DE ACTIVIDAD PROFESIONAL}

Al evocar la presencia francesa en el Perú hacemos sobre todo referencia a algunas personalidades que han marcado de una u otra manera la historia y la sociedad peruanas. Pensamos en particular en aquellos hombres de negocios muy involucrados en la historia económica del país (como Thomas Lachambre o Auguste Dreyfus), en aquellos expertos contratados por el gobierno peruano para tomar las riendas — incluso crear - de instituciones administrativas, científicas o universitarias (el estadístico Marchand, el agrónomo Jean-Baptiste Henri Martinet, el jurista Pradier-Fodéré), los arquitectos (Mimey, Chalon, etc.), los ingenieros enviados por Gustave Eiffel, los pintores y fotógrafos (Courret, Garreaud y tantos otros mucho menos conocidos). Aunque ellos marcaron su época o son más visibles, es conveniente observar que ellos constituyen desde luego una pequeña minoría 26 en relación al conjunto de franceses que vinieron a instalarse -a veces solo por algunos años, a veces definitivamente- al Perú.

La distribución de los franceses por grandes sectores de actividad, tal como se puede ver a través de los registros del consulado, subraya la alta predominancia de artesanos ( $28 \%$ del total de los individuos que aparecen en los registros, $33 \%$ de los activos cuya profesión se conoce). En primer lugar, se observará que entre ellos hay un gran número de artesanos especializados en el trabajo de la madera (104 carpinteros, 68 carpinteros de armar, 44 ebanistas), así como en otras actividades ligadas a la construcción (pintores de brocha gorda, talladores de piedra, cerrajeros, albañiles), también los oficios vinculados al hierro (herrero, carretero, calderero, hojalatero). En el rubro de vestido y adornos hay evidentemente un número considerable de artesanos franceses (sombrereros, sastres, costureras, zapateros, tintoreros, peluqueros y joyeros). Por último, confirmando la reputación francesa por la comida, en particular panaderos y pasteleros (72 artesanos, o sea casi $7 \%$ de ellos), carniceros (35) y charcuteros (7) y finalmente confiteros (11). Cuando a mediados del siglo XIX la emigración europea comenzó a hacerse importante, el aflujo de artesanos respondió de cierta manera a la evolución del gusto y de las costumbres de consumo (gusto por el lujo y los productos «a la europea»). Según Gootenberg (1990: 245), sin duda estos artesanos favorecieron la introducción de productos manufacturados europeos (introducidos subrepticiamente en la producción local de los artesanos europeos asentados en el Perú) así como materias primas europeas.

El segundo gran sector representado es el de los comerciantes (con $24,5 \%$ del total de los inscritos y $29 \%$ de los activos - cifra a la que hay que agregar una parte de los individuos inscritos en la categoría «empleados»—). Si entre ellos hay algunas personalidades ligadas al mundo internacional de los negocios, así como algunos otros que pueden ser considerados como negociantes de respetable importancia, la mayoría de los franceses incluidos en la categoría de comerciantes se dedica más bien al comercio minorista. La especialidad de los comerciantes es muy poco conocida; cuando aparece, se trata a menudo de comerciantes vinculados al rubro del vestido, de la moda, de los adornos; cabe citar también a los libreros, a los armeros y a los tenderos. A este gran conjunto relacionado con las actividades comerciales se le puede también añadir un sector específico: el de la hotelería y de los restaurantes. No asombra, sin duda, constatar que este sector está ampliamente representado (con 6,7 \% de los activos, a los que se puede agregar una parte de los empleados de servicio27). iEn el registro encontramos 147 cocineros! (pero no necesariamente todos trabajaban en un restaurante u hotel). Cabe señalar que algunos hoteles de origen francés pertenecen a la historia de la Lima republicana, como el hotel Maury o el hotel Morin.

alsacianos y los lorenos residentes ya en América también fueron invitados a optar por una u otra nacionalidad: en el caso del Perú encontramos los nombres de 5 personas (Poivey \& Ganter, 1990).

26 Los franceses que ocuparon funciones importantes (ejecutivos y administradores, profesores, médicos, industriales, ingenieros) o valorizantes (artistas o grandes artesanos) representan aproximadamente $10 \%$ del conjunto de los activos.

27 Las mucamas y las lavanderas, por ejemplo, pueden también haber trabajado para un hotel o en casas particulares. No es siempre fácil establecer categorías claramente definidas. 
Para dar término al tema relacionado con las actividades profesionales ocupadas por los migrantes franceses mencionaremos la presencia importante de personas vinculadas a los oficios agrícolas ( $4 \%$ de los activos; aquí encontramos en particular a labradores pero también a jardineros y ya a viticultores), a obreros (3\% de los activos; los mecánicos están ampliamente representados) y a marinos ( $2 \%$ de los activos identificados).

Si hablamos de emigración «discreta», es porque en primer lugar la emigración francesa con destino al Perú es un fenómeno totalmente menor en la historia de los movimientos migratorios franceses observada en su conjunto: no ha habido propiamente hablando emigración organizada, como sí la hubo para Uruguay o para la Argentina, en cuyo caso se ha identificado a un cierto número de agencias de emigración que trabajaban en Francia para reclutar candidatos a la colonización. Las partidas con destino al Perú siempre fueron espontáneas, ya sea por la imagen que se hacían del Perú o por el éxito de un pariente o vecino o de alguna vaga relación en su pueblo o en otro pueblo del cantón. Por otro lado, si algunas individualidades o ciertas actividades profesionales o comerciales contribuyeron mucho a no dudarlo en alimentar la reputación de Francia en el Perú (ya se trate de tal o cual profesor, administrador, ingeniero, artista, hotelero u hombre de negocios), las personas aquí concernidas siempre constituyeron una escasa minoría en relación al conjunto de los franceses que vinieron a asentarse al Perú. Si las actividades profesionales de algunos artesanos o comerciantes pudieron a veces suscitar reacciones proteccionistas, incluso hostiles, por parte de sus homólogos peruanos o de corporaciones nacionales, estos son solo epifenómenos muy circunscritos en el tiempo y en el espacio. Más bien, la comunidad francesa parece haber sido bien aceptada y haberse integrado a su nueva tierra de adopción. Si bien no todos los franceses escogieron permanecer definitivamente en el Perú, generalmente aquellos que lo hicieron se «fundieron» en el crisol peruano. A tal punto que si no hubiésemos dispuesto de los registros del Consulado de Francia, no habría sido fácil notar esta presencia francesa y analizar sus particularidades.

\section{Referencias citadas}

BARRANTES RODRÍGUEZ LARRAÍN, F., 2005 - Banderas de la libertad. Militares franceses en la Independencia del Perú, 93 p.; Lima: Sociedad Francesa de Beneficencia.

BLANCPAIN, J.-P., 1999 - Le Chili et la France (XVIII'-XXe siècles), 240 p.; París: L'Harmattan.

BONFIGLIO VOLPE, G., 1987 - Introducción al estudio de la inmigración europea en el Perú. In: Primer seminario sobre poblaciones inmigrantes, tomo 1 (Wilfredo Kapsoli et al., eds.): 31-78; Lima: Consejo Nacional de Ciencia y Tecnología.

BONFIGLIO, G., 2001 - La presencia europea en el Perú, 381 p.; Lima: Fondo Editorial del Congreso de la República.

BONNAIN, R., 2000 - Migrations et inscription urbaine des Pyrénées en Amérique du Sud au XIXe siècle : Montevideo et Caracas. Annales de démographie historique, $\mathbf{n}^{\circ} 1$ : 61-76.

BOURDÉ, G., 1974 - Urbanisation et immigration en Amérique latine, 288 p.; Buenos AiresParís: Editions Aubier-Montaigne.

CAMPBELL, L.G., 1972 - The foreigners in peruvian society during the eighteenth century. Revista de Historia de América, $\mathbf{n}^{\circ}$ 73-74: 153-163; México: Instituto panamericano de geografía e historia.

CHARNISAY, H. de, 1996 - Émigration basco-béarnaise en Amérique, 270 p.; Biarritz: J\&D Éditions.

DAHLGREN, E.W., 1909 - La France et les côtes de l'océan Pacifique, 740 p.; París: Librairie ancienne Honoré Champion.

GOOTENBERG, P., 1990 - Los origenes sociales del proteccionismo y libre comercio en Lima del siglo XIX. Histórica, vol. XIV, $\mathbf{n}^{\circ}$ 2, dic.: 235-279. 
Los franceses en el Perú en el siglo XIX: retrato de una emigración discreta

HAMPE MARTÍNEZ, T., 1993 - Apuntes documentales sobre inmigrantes europeos y norteamericanos en Lima (siglo XIX). Revista de Indias, LIII, $\mathbf{n}^{\circ}$ 198: 459-491.

HÜNEFELDT, C., 1987 - Inserción socioeconómica de los extranjeros en el Perú... In: Primer seminario sobre poblaciones inmigrantes, tomo I, 234 p.; Lima: Consejo Nacional de Ciencia y Tecnología.

LA BARBINAIS, Le Gentil de, 1728 - Nouveau voyage autour du monde; Amsterdam: chez Pierre Mortier, vol. I.

LAFOND DE LURCY, G., 1844 - Voyages autour du monde et naufrages célèbres. Voyages dans les Amériques, Vol. 2, 432 p.; París: Administration de librairie.

LESPAGNOL, A., 1997 - Messieurs de Saint-Malo. Une élite négociante au temps de Louis XIV, 2 vols., 867 p.; Rennes: Presses universitaires de Rennes, II.

POIVEY, J.-P. \& GANTER, A., 1990 - Les Alsaciens-Lorrains ayant opté pour la nationalité française en 1872. Secteur géographique : Amérique du Sud, 10f.; Vesoul et Morschwiller-le-Bas.

RIVIALE, P., 2005 - Un négociant français au Pérou au XIXe siècle : splendeur et décadence de Félix Dibos (1831-1898). HISAL, http/hisal.org/vol.1.

ROUDIÉ, P., 1983-1984 - Bordeaux, port d'émigration lointaine (1865-1918). Revue historique de Bordeaux et du département de la Gironde, tome XXX, nouvelle série: 157-188.

SOCIEDAD FRANCESA DE BENEFICENCIA, 2002 - Memoria institucional, 272 p.; Lima.

TARDIEU, J.-P., 1995 - L'Inquisition de Lima et les hérétiques étrangers. XVI-XVIle siècles, 176 p.; París: L'Harmattan.

WITT, H., 1992 - Diario 1824-1890. Un testiminio personal sobre el Perú del siglo XIX. Volumen 1 (1824-1842), 576 p.; Lima: Banco Mercantil. 